\title{
On the Sensitivity of BDDs with Respect to Path-Related Objective Functions
}

\author{
Rüdiger Ebendt Rolf Drechsler \\ Institute of Computer Science \\ University of Bremen \\ 28359 Bremen, Germany \\ Email: \{ebendt,drechsle\}@informatik.uni-bremen.de
}

\begin{abstract}
Reduced ordered Binary Decision Diagrams (BDDs) are a data structure for efficient representation and manipulation of Boolean functions. They are frequently used in logic synthesis and formal verification. In recent practical applications, BDDs are optimized with respect to new objective functions.

In 1986, Bryant showed that, dependent on a chosen variable ordering, the size of BDDs can vary from linear to exponential. In this paper we derive similar results for the sensitivity of BDDs with respect to path-related objective functions. First, we provide a theoretical view, giving examples for the large variation in the maximal path length and the expected path length in BDDs. This shows how important it is to choose a good or optimal variable ordering.
\end{abstract}

Experimental results show the sensitivity of benchmark functions with respect to all considered objective functions.

\section{INTRODUCTION}

Reduced ordered Binary Decision Diagrams (BDDs) were introduced in [1] and are well-known from logic synthesis and hardware verification.

Run time and space requirement of BDD-based algorithms depend on the size of the BDD. However, this size is very sensitive to a chosen variable ordering [1]: dependent on a chosen variable ordering, the size of BDDs can vary from linear to exponential. In general, determining an optimal variable ordering is a difficult problem. It has been shown that it is NP-complete to decide whether the number of nodes of a given BDD can be improved by variable reordering [2]. For this reason, many heuristic methods to determine a good ordering have been proposed, based on structural information [3] or on dynamic reconstruction [4].

Similar questions arise for alternative, path-related objective functions. The optimization with respect to the number of paths in a BDD has been studied in [5]: the number of paths in a circuit derived from a BDD corresponds to the number of paths in the BDD. It is proportional to the number of faults under the path delay fault model. Hence minimizing the number of paths can significantly reduce the time for testing BDD circuits [6]. It also can be used for minimizing Disjoint-Sum-Of-Products (DSOPs) [7] which are used in the calculation of spectra of Boolean functions [8] or as starting point for the minimization of Exclusive-SumOf-Products (ESOPs) [8]: in a BDD for a Boolean function $f$, each path to the 1-terminal corresponds to a (partial) assignment to the variables, i.e. to a product of the literals of $f$. The products derived from different paths are disjoint. Collecting them in a sum yields a DSOP. The optimization with respect to the Expected Path Length (EPL) has been studied in [9], [10]. It is motivated by the reduction of the time needed to evaluate many test vectors using BDDs in functional simulation [11], [12]. Minimization of EPL as well as of the Maximal Path Length (MPL) in BDDs is also motivated by logic synthesis: first, every variable missing in a path of the BDD corresponds to a don't care. Thus shortening the EPL can help providing don't care values for minimization. Second, the longest path in the BDD corresponds to the critical path in a derived circuit. Hence minimization with respect to MPL/EPL is expected to support synthesis approaches targeting the delay of the resulting circuits [13]. The minimization of MPL has been studied in [10], [14].

Good run times of all these applications can only be achieved if the BDDs are optimized with respect to the new objective functions. Hence it is crucial to understand in how far these objectives depend on the variable ordering, i.e. the sensitivity of the new objective functions must be studied.

This paper first gives a theoretical study of the sensitivity of path-related objective functions for BDDs. For each criterion, the sensitivity is stated as the largest known ratio of the objective functions value for the worst-case and the bestcase BDD for one and the same function. The results are constructive as example BDDs with the stated sensitivity are given.

Experimental results give the discussed sensitivities for benchmark functions. For this purpose, BDDs have been maximized, then minimized with respect to the respective objective functions and finally the resulting ratios have been computed. The experiments clearly show a high sensitivity of the benchmark functions. This demonstrates how important it is to choose a good ordering during the respective applications.

\section{BACKGROUND}

Reduced ordered Binary Decision Diagrams (BDDs) are directed acyclic graphs where a Shannon decomposition

$$
f=x_{i} f_{x_{i}}+\bar{x}_{i} f_{\bar{x}_{i}} \quad(1 \leq i \leq n)
$$

is carried out with each node. Each node $v$ is labeled with a Boolean variable in $\left\{x_{1}, \ldots, x_{n}\right\}$. The variables are encountered at most once and in the same order, the "variable ordering" denoted $\pi$, on every path from the root node to one of the two terminal nodes. For a BDD $F$, a prefix $\pi$ (e.g. $\pi F$ ) expresses that $F$ respects the ordering $\pi$.

Note that reduced diagrams are considered, derived by removing redundant nodes and merging isomorphic subgraphs. In the following we assume shared BDDs with Complement 
Edges (CEs) [15] without mentioning it further (and without using CEs in the illustrations). Note that all results reported here directly transfer to BDDs without CEs. The root nodes of a BDD always have external references, i.e. they represent user functions. Together with other nodes representing user functions they constitute the set of output nodes. For more details see [1].

Paths in a BDD start at a root node and end at a terminal node. The length of a path is the number of inner nodes on the path. Next, path-related objective functions are defined: the EPL of a BDD expresses the expected number of variable tests needed to evaluate an input assignment along a path from an output node to a terminal node. This number is determined as the average path length under all such input assignments. For a BDD $F$ it is denoted $\epsilon(F)$. For a BDD node $v, \epsilon(v)$ is the EPL of the sub-BDD rooted at $v$. In case of a single-rooted BDD $F$, the EPL is simply the $\epsilon$-value of the root node, otherwise it is the average of the weighted ${ }^{1} \epsilon$-values for all output nodes. Another path-related objective function for BDDs is the MPL: let $\mu(F)$ denote the maximal length of a path from an output node to a terminal node.

\section{RELATED WORK}

It is well-known that the size of BDDs is often very sensitive to a chosen variable ordering. In [1] an example has been given where the BDD size varies from linear to exponential dependent on the ordering of the variables (see Fig. 1). An analogous result on the sensitivity of the number of paths in BDDs has been given in [5]. Our work extends the scope of these results to other path-related criteria, namely expected and maximal path length. Recently, in [16] a study on the Average Path Length $\left(\mathrm{APL}^{2}\right)$ in BDDs states best APLs under all orderings for specific functions (e.g. $n$-input AND/OR) and compares the expected value of APLs for classes of functions to the maximum APL for each class. In contrast, our study focusses on the largest observable ratio of maximum and minimum EPL for one and the same function.

\section{Sensitivity}

In this section, we give results similar to [1], [5] for the sensitivity of the EPL and MPL in BDDs: in essence, there are $n$-ary BDD functions for which the EPL under different orderings varies by a factor of $\Theta(n)$. On the other hand, the variation of the MPL for certain BDD functions under different orderings still is up to a factor of $\Theta(\sqrt{n})$. This shows how important it is to determine a good ordering for the new pathrelated objective functions.

Lemma 1: Let $f: \mathbf{B}^{n^{2}} \rightarrow \mathbf{B}$ be defined as

$$
f=\sum_{i=1}^{n} \prod_{k=1}^{i-1} \bar{x}_{k} \cdot \prod_{k=0}^{n-1} x_{k \cdot n+i} .
$$

Let $F$ be a BDD representing $f$ and let the variable orderings

\footnotetext{
${ }^{1}$ The weight equals the number of external references to the output node.

${ }^{2}$ In the case of single-output functions, the APL coincides with the EPL.
}

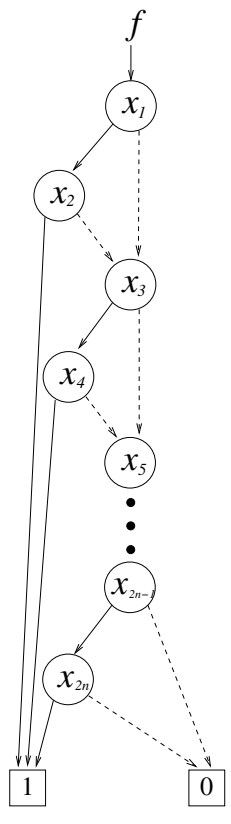

(a) EPL is bounded by the constant 6 .

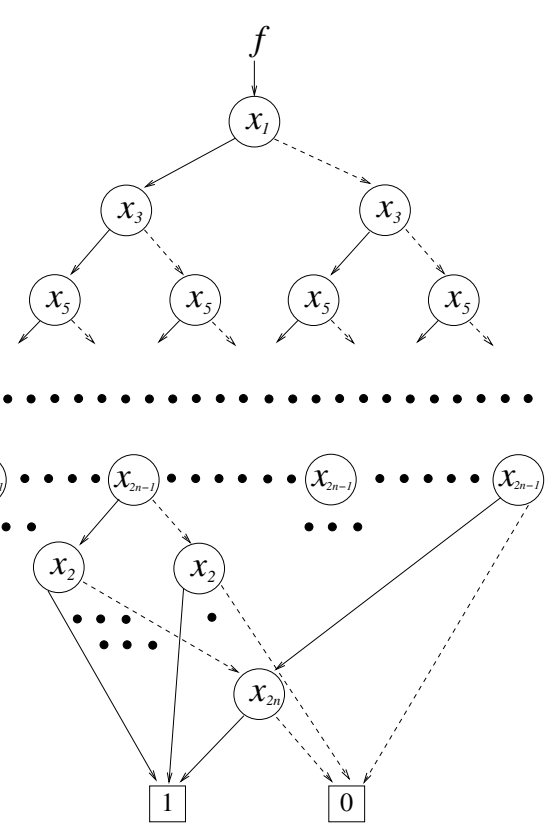

(b) EPL is greater than $n$.
Fig. 1. Two BDDs for $f=x_{1} \cdot x_{2}+x_{3} \cdot x_{4}+\ldots+x_{2 n-1} \cdot x_{2 n}$.

$\pi_{1}, \pi_{2}$ be given as

$$
\begin{aligned}
\pi_{1}= & x_{1}, x_{n+1}, \ldots, x_{(n-1) \cdot n+1}, x_{2}, x_{n+2}, \ldots, \\
& x_{(n-1) \cdot n+2}, \ldots, x_{n}, x_{2 \cdot n} \ldots, x_{n^{2}} \text { and } \\
\pi_{2}= & x_{(n-1) \cdot n+1}, x_{(n-2) \cdot n+1}, \ldots, x_{1}, x_{(n-1) \cdot n+2}, \\
& x_{(n-2) \cdot n+2}, \ldots, x_{2}, \ldots, x_{n^{2}}, x_{(n-1) \cdot n}, \ldots, x_{n} .
\end{aligned}
$$

It is

$$
\begin{aligned}
& \mu\left(\pi_{1} F\right)=2 \cdot n-1, \text { and } \\
& \mu\left(\pi_{2} F\right)=n^{2} .
\end{aligned}
$$

The BDD $\pi_{1} F$ is shown in Fig. 2(a). As illustrated in Fig. 2(b) it can be transformed into $\pi_{2} F$ by a series of function-preserving variable swaps [17], [4]. This results in the BDD given in Fig. 2(c). Along the thickened edges, the longest paths traverse $2 \cdot n-1$ and $n^{2}$ edges, respectively.

Lemma 2: Let $f: \mathbf{B}^{2 \cdot n} \rightarrow \mathbf{B} ;\left(x_{1}, x_{2}, \ldots, x_{2 n}\right) \mapsto x_{1} \cdot x_{2}+$ $x_{3} \cdot x_{4}+\ldots+x_{2 \cdot n-1} \cdot x_{2 \cdot n}$. Let the variable orderings $\pi_{1}, \pi_{2}$ be given as

$$
\begin{aligned}
& \pi_{1}=x_{1}, \ldots, x_{2 \cdot n}, \text { and } \\
& \pi_{2}=x_{1}, x_{3}, \ldots, x_{2 \cdot n-1}, x_{2}, x_{4}, \ldots, x_{2 n} .
\end{aligned}
$$

It is

$$
\begin{aligned}
& \epsilon\left(\pi_{1} F\right)<6, \text { and } \\
& \epsilon\left(\pi_{2} F\right)>n .
\end{aligned}
$$

The BDD $\pi_{1} F$ is shown in Fig. 1(a). Recently, a similar result has been shown for the APL by the use of generating functions and differentiation [16] which is not needed in our proof: using a recurrent definition of $\epsilon(v)$ [18], $\epsilon\left(\pi_{1} F\right)$ can be expressed as a geometric series with the closed form $6 \cdot\left(1-\left(\frac{3}{4}\right)^{n}\right)$. The BDD $\pi_{2} F$ is shown in Fig. 1(b). Along all paths from the root node to the terminal nodes, at least the $n$ variables with an odd subscript are tested. Hence, $\epsilon\left(\pi_{2} F\right)>n$ follows. 


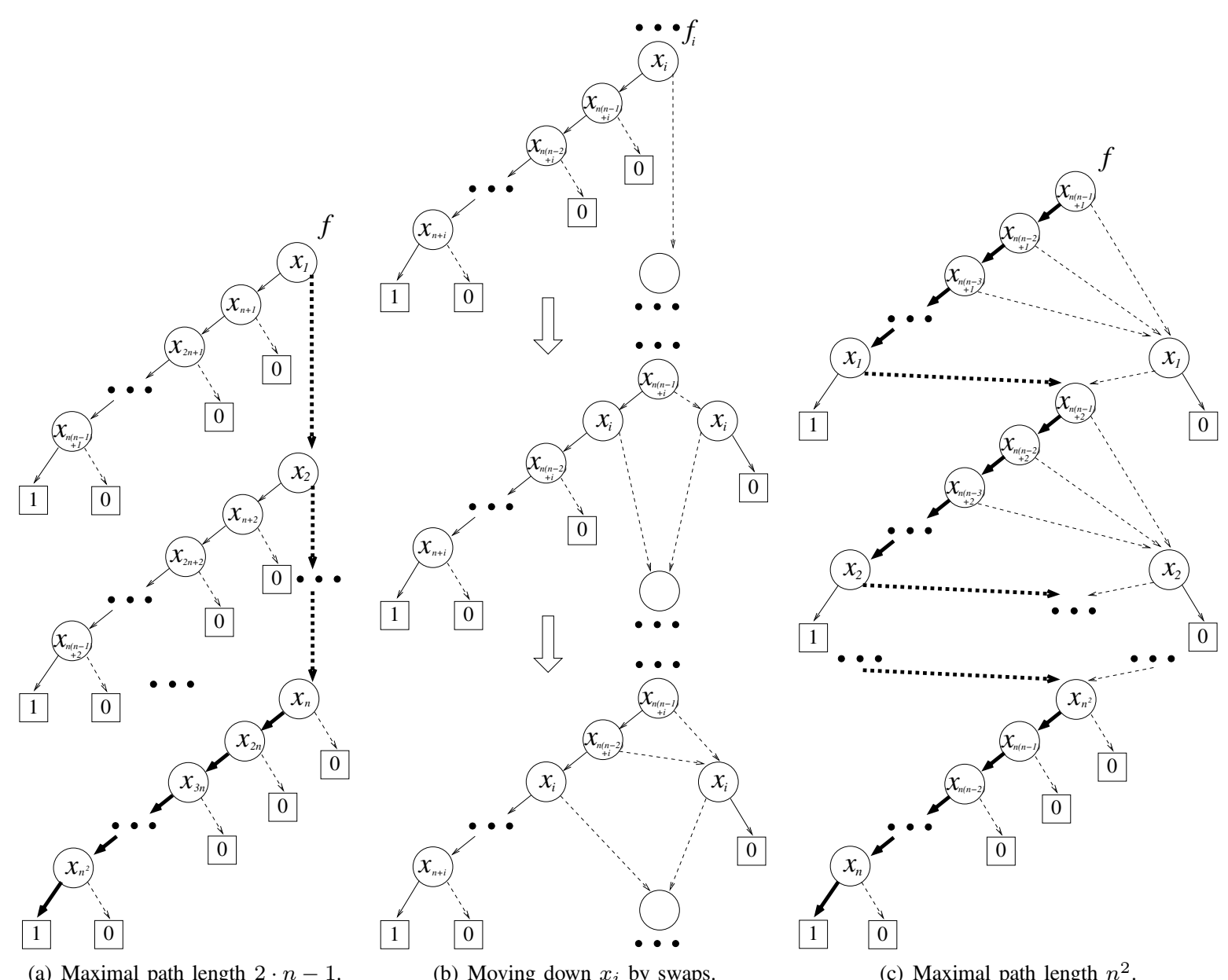

Fig. 2. A worst-case example for the sensitivity of the MPL in BDDs.

\section{EXPERIMENTAL RESULTS}

In this section we present the results of an experimental study on the sensitivity of benchmark functions. From the logic level description of the circuits of the LGSynth93 benchmark set [19], BDDs have been built. Afterwards, the variable ordering of the initial BDDs has been altered in order to maximize or minimize the following objective functions: MPL, EPL, and the number of paths in BDDs. For this purpose, the sifting approaches as given in [10] have been used. Note that these approaches do not yield exact solutions as they are faster heuristic approaches based on dynamic reordering [4]. Obviously, the minimization methods can also be used for maximization with only minor changes.

To put up a testing environment, all algorithms have been integrated into the CUDD package [20] and were tested in the same system environment. We used an AMD64 3500+ system with a CPU running at $2.2 \mathrm{GigaHz}$ with a main memory of 1 GigaByte for our experiments. A time limit of $36,000 \mathrm{CPU}$ seconds has been applied. Due to space limitation and since we are only interested in the sensitivity, no computation times or memory requirements of the algorithms are given here. Instead we refer to [10] for detailed information about the used sifting modifications.

In a series of experiments, the sensitivity of BDDs for benchmark functions with respect to the three objective func- tions has been determined. The results are given in Table I. In three cases the run time of the optimization exceeded the predefined time limit. The numbers in brackets give the last (highest) observed values for these cases. In the first column the name of the function is given. Column in denotes the number of inputs of a function. The next three multi-columns MPL, EPL, and \# paths give the maximal/minimal observed function values of the objective functions MPL, EPL and number of paths in BDDs, respectively, as well as the ratio of maximal and minimal value, i.e. the sensitivity. Each multicolumn consists of the corresponding three sub-columns max, min, and sens., respectively.

As the results show, the sensitivity of the considered functions stays below the upper bounds stated in Section IV. However, still variations as high as a factor of two can be observed (e.g., see dalu). Similar results have been obtained for the other objective functions: the variation in EPL can be as high as a factor of five (e.g., see dalu). For the number of paths the theory states the existence of exponential ratios. This is confirmed by the experiments: six benchmark functions (c3540, c880, dalu, $k 2$, rot, s838.1) show sensitivities of several orders of magnitudes (e.g., for $s 838.1$ a sensitivity of $1.8 \cdot 10^{12}$ has been observed). 
TABLE I

SENSITIVITY OF BENCHMARK FUNCTIONS WITH RESPECT TO DIFFERENT OBJECTIVE FUNCTIONS

\begin{tabular}{|c|c|c|c|c|c|c|c|c|c|c|}
\hline \multirow[t]{2}{*}{ name } & \multirow[t]{2}{*}{ in } & \multicolumn{3}{|c|}{ MPL } & \multicolumn{3}{|c|}{ EPL } & \multicolumn{3}{|c|}{ \# paths } \\
\hline & & $\max$ & $\min$ & sens. & $\max$ & $\min$ & sens. & $\max$ & $\min$ & sens. \\
\hline$\overline{\text { apex6 }}$ & 135 & 24 & $\overline{20}$ & $\overline{1.2}$ & $\overline{44.87}$ & $\overline{2.33}$ & $\overline{2.09}$ & $\overline{105935}$ & $\overline{822}$ & $\overline{128.87}$ \\
\hline apex7 & 49 & 24 & 19 & 1.26 & 6.33 & 2.25 & 2.81 & 97067 & 605 & 160.44 \\
\hline b9 & 41 & 14 & 13 & 1.07 & 4.36 & 2.65 & 1.65 & 1109 & 245 & 4.53 \\
\hline c1355 & 41 & 41 & 41 & 1.00 & 35.18 & 20.82 & 1.72 & (1308723511296) & 336846811136 & (3.89) \\
\hline c3540 & 50 & (39) & 30 & $(1.30)$ & (20.88) & 9.81 & (2.13) & 289570968908 & 5448959 & 53142.44 \\
\hline c499 & 41 & 41 & 41 & 1.00 & 34.93 & 20.34 & 1.72 & 1350272286720 & 351879196672 & 3.84 \\
\hline c880 & 60 & 45 & 41 & 1.10 & 11.50 & 4.82 & 2.39 & 327753942183 & 1034704 & 316761.07 \\
\hline cht & 47 & 6 & 4 & 1.5 & 3.15 & 2.06 & 1.53 & 124 & 81 & 1.53 \\
\hline dalu & 75 & (47) & 24 & (1.96) & 26.40 & 4.94 & 5.34 & 9697774296 & 2298 & 4220093.25 \\
\hline example2 & 85 & 16 & 14 & 1.14 & 4.33 & 2.18 & 1.99 & 1621 & 384 & 4.22 \\
\hline frg2 & 143 & 25 & 20 & 1.25 & 6.61 & 2.32 & 2.85 & 142061 & 5846 & 24.30 \\
\hline i3 & 132 & 32 & 32 & 1.00 & 12.49 & 4.46 & 2.80 & 262148 & 262148 & 1.00 \\
\hline i5 & 133 & 19 & 19 & 1.00 & 7.00 & 1.98 & 3.54 & 22453 & 370 & 60.68 \\
\hline i6 & 138 & 5 & 4 & 1.25 & 3.99 & 3.05 & 1.31 & 486 & 270 & 1.80 \\
\hline i9 & 88 & 13 & 12 & 1.08 & 10.88 & 4.96 & 2.19 & 92470 & 2320 & 39.86 \\
\hline k2 & 45 & 39 & 24 & 1.63 & 12.23 & 4.01 & 3.07 & 3527535452 & 1481 & 2381860.53 \\
\hline rot & 107 & 60 & 57 & 1.05 & 7.61 & 3.08 & 2.47 & 5898224798799 & 17824477 & 330905.91 \\
\hline s641 & 54 & 27 & 25 & 1.08 & 6.30 & 2.65 & 2.38 & 72066 & 1700 & 42.39 \\
\hline s713 & 54 & 27 & 25 & 1.08 & 4.50 & 2.65 & 1.69 & 72066 & 1700 & 42.39 \\
\hline s838.1 & 66 & 66 & 38 & 1.73 & 4.34 & 2.92 & 1.49 & 1104017058104789 & 593 & 1861748833228.99 \\
\hline $\mathrm{x} 1$ & 51 & 23 & 22 & 1.04 & 5.09 & 2.67 & 1.90 & 62817 & 1553 & 40.45 \\
\hline $\mathrm{x} 3$ & 135 & 24 & 20 & 1.20 & 4.88 & 2.34 & 2.09 & 126320 & 916 & 137.90 \\
\hline $\mathrm{x} 4$ & 94 & 15 & 15 & 1.00 & 4.72 & 2.39 & 1.97 & 3794 & 687 & 5.52 \\
\hline
\end{tabular}

\section{CONCLUSION}

Recent practical applications have raised the discussion of new path-related objective functions for BDDs.

In this paper, the sensitivity of BDDs to variable ordering with respect to path-related objective functions has been investigated. A result of Bryant, 1986, has been extended to the new objective functions. Examples for the large variation of the maximal path length and the expected path length, dependent on the chosen variable ordering, have been given. Experiments show that BDDs for benchmark functions can be very sensitive for all optimality criteria, confirming the obtained theoretical results.

\section{REFERENCES}

[1] R. E. Bryant, "Graph-based algorithms for Boolean function manipulation," IEEE Trans. on Comp., vol. 35, no. 8, pp. 677-691, 1986.

[2] B. Bollig and I. Wegener, "Improving the variable ordering of OBDDs in NP-complete," IEEE Trans. on Comp., vol. 45, no. 9, pp. 993-1002, 1996.

[3] H. Fujii, G. Ootomo, and C. Hori, "Interleaving based variable ordering methods for ordered binary decision diagrams," in Int'l Conf. on CAD 1993, pp. 38-41.

[4] R. Rudell, "Dynamic variable ordering for ordered binary decision diagrams," in Int'l Conf. on CAD, 1993, pp. 42-47.

[5] G. Fey and R. Drechsler, "Minimizing the number of paths in BDDs theory and algorithm," To appear in IEEE Trans. on CAD 2005, preliminary version in 15th Symp. on Integrated Circuits and System Design, pp. 359-364, 2002.

[6] R. Drechsler, J. Shi, and G. Fey, "Synthesis of fully testable circuits from BDDs," IEEE Trans. on CAD, vol. 23, no. 3, pp. 440-443, 2004.

[7] G. Fey and R. Drechsler, "Utilizing BDDs for disjoint SOP minimization," in 45th IEEE International Midwest Symp. on Circuits and Systems, 2002, pp. 306-309.

[8] M. Thornton, R. Drechsler, and D. Miller, Spectral Techniques in VLSI CAD. Kluwer Academic Publisher, 2001.
[9] S. Nagayama, A. Mishchenko, T. Sasao, and J. Butler, "Minimization of average path length in BDDs by variable reordering," in Proc. of International Workshop on Logic and Synthesis, 2003.

[10] R. Ebendt, W. Günther, and R. Drechsler, "Minimization of the expected path length in BDDs based on local changes," in Asian and South Pacific Design Automation Conf., 2004, pp. 866-871.

[11] P. Ashar and S. Malik, "Fast functional simulation using branching programs," in Int'l Conf. on CAD, 1995, pp. 408-412.

[12] Y. Jiang, S. Matic, and R. Brayton, "Generalized cofactoring for logic function evaluation," in Design Automation Conf., 2003, pp. 155-158.

[13] C. Scholl and B. Becker, "On the generation of multiplexer circuits for pass transistor logic," in Design, Automation and Test in Europe, 2000, pp. 372-378.

[14] S. Nagayama and T. Sasao, "On the minimization of longest path length for decision diagrams," Proc. of International Workshop on Logic and Synthesis, pp. 28-35, 2004

[15] K. Brace, R. Rudell, and R. Bryant, "Efficient implementation of a BDD package," in Design Automation Conf., 1990, pp. 40-45.

[16] J. Butler, T. Sasao, and M. Matsuura, "Average path length of binary decision diagrams," IEEE Trans. on Comp., vol. 54, no. 9, September 2005.

[17] N. Ishiura, H. Sawada, and S. Yajima, "Minimization of binary decision diagrams based on exchange of variables," in Int'l Conf. on CAD, 1991, pp. $472-475$.

[18] Y. Y. Liu, K. H. Wang, T. T. Hwang, and C. L. Liu, "Binary decision diagram with minimum expected path length," in Design, Automation and Test in Europe, 2001, pp. 708-712.

[19] Collaborative Benchmarking Laboratory, "1993 LGSynth Benchmarks," North Carolina State University, Department of Computer Science, 1993.

[20] F. Somenzi, “CU Decision Diagram Package Release 2.4.0,” University of Colorado at Boulder, 2004. 